\title{
Como identificar a originalidade num artigo científico ou numa tese de doutoramento?
}

\section{How to identify originality in a scientific manuscript or in a doctoral thesis?}

\author{
João Paulo Vagarinho*
}

\begin{abstract}
RESUMO
Originalidade é um requisito indispensável na ciência, principalmente para a aceitação de teses de doutoramento ou quando se pretende publicar artigos científicos. $\mathrm{Na}$ literatura existente, podemos encontrar muitas definições sobre originalidade, porém não existe um consenso alargado para adotar uma definição generalizada; como resultado, verifica-se falta de rigor nas definições que envolvem o termo, falta de clareza sobre a identificação das suas características, ausência de uma descrição sobre elas e não conseguimos entender quais as características que diferem um artigo cientifico de uma tese de doutoramento. Recorrendo a dois inquéritos construídos para o efeito e à revisão bibliográfica de 48 artigos durante 2017, encontramos 12 características que são específicas e concretas do termo originalidade. Em adição, encontrámos o que difere o termo originalidade quando este está ligado a um artigo cientifico e a uma tese de doutoramento. Assim, já existe uma base para investigadores, professores, equipas editoriais e alunos poderem identificar a originalidade em trabalhos científicos ou para outras partes interessadas construírem uma definição correta para este termo. $\mathrm{O}$ trabalho apresentado é igualmente benéfico em contextos de estandardização e qualidade da investigação cientifica.
\end{abstract}

Palavras-chave: Originalidade. Artigo cientifico. Tese de doutoramento. Dissertação.

\footnotetext{
ABSTRACT

Originality is an indispensable requirement in science, especially for the acceptance of doctoral theses or when one intends to publish scientific articles.

* Universidade de Vigo. Vigo, Pontevedra, Espanha. E-mail: joao.paulo.vagarinho@gmail. com. https://orcid.org/0000-0003-0084-7810.
} 
In the existing literature, we can find many definitions of originality, but there is no broad consensus to adopt a generalized definition; as a result, there is a lack of rigor in definitions involving the term, lack of clarity about the identification of their characteristics, lack of a description about them and we can't understand the characteristics that differ a scientific article from a doctoral thesis. Using two surveys constructed for this purpose and the bibliographic review of 48 scientific articles during 2017, we found 12 characteristics that are specific and concrete of the term originality. In addition, we found what differs the term originality when it is linked to a scientific article and a doctoral thesis. Thus, there is already a basis for researchers, teachers, editorial teams and students to be able to identify originality in scientific work or for other stakeholders to construct a correct definition for this term. The work presented is also beneficial in contexts of standardization and quality of scientific research..

Keywords: Originality. Scientific manuscript. Ph.D. Thesis. Dissertation.

\section{Introdução}

Originalidade é uma componente que integra a qualidade da investigação cientifica (Mårtenssona, Forsb, Wallinc, Zanderd, \& Nilsson, 2016), nomeadamente na aceitação de teses de doutoramento (Kyvik \& Thune, 2014) e na publicação de artigos científicos (Peng, 2015). Por esta razão, analisamos um conjunto de artigos que estão ligados a estes temas e que fazem referência ao termo "originalidade". Todos os artigos referem que o tema "originalidade" é de grande importância, principalmente no momento de o identificar em qualquer trabalho cientifico, mas nunca explicam em concreto e detalhadamente o seu significado de forma abrangente; as suas caraterísticas, como as identificamos ou qual a diferença entre originalidade num artigo cientifico e numa tese de doutoramento, são exemplos da ausência dessa identificação os artigos escritos por Torrance et al. (1993), Zuber-Skerritt \& Fletcher (2007) ou Lundgren \& Robertsson (2013). Outros artigos, relativos ao mesmo tema, nem fazem referência a "originalidade" como requisito para publicar artigos científicos e/ou na aceitação de teses de doutoramento, este parece ser um fator ignorado, como é o caso do artigo escrito por Brooks \& Normore (2015) e do artigo escrito por Harwood (2005). Por outro lado, em algumas universidades, existe maior preocupação em que os estudantes optem por uma análise intelectual rigorosa (Serenko, Bontis, Booker, Sadeddin, \& Hardie, 2010), crítica (Watts, 2008), resolvam problemas ou apliquem conhecimentos em novas situações (Jonassen, 2000) do que propriamente mencionem explicitamente a originalidade (Bourke \& Holbrook, 
2011). Em outros artigos analisados, verificou-se que um grupo de autores afirmam que originalidade é um julgamento subjetivo (Pieters, Warlop, \& Wedel, 2002) e (Silvia, Martin, \& Nusbaum, 2009), outros realçam que definir originalidade é difícil (Stigler, 1995) e outros ainda afirmam que originalidade não é algo suficientemente interessante para garantir uma publicação cientifica (Coniam, 2012). Talvez a falta de rigor na definição ou a falta de um conjunto de características encoraje alguns autores a reclamar sobre a dificuldade que sentem em trabalhar com alunos com o tema originalidade (Runco, 2015). Existem também autores que escrevem no mesmo artigo sobre dois temas, que são criatividade e originalidade, contudo, pela ausência de definições abrangentes e claras, esses artigos podem conduzir os leitores a interpretações pouco esclarecedoras ou até confusas (Uidhir \& Freeland, 2015). Como ponto de partida, poderemos dizer que criatividade é a capacidade cognitiva para fazer associações e combinar categorias amplas ou muitas ideias simultaneamente (Cropley, 2000), é pensar em algo de novo, valioso e aberto que nos ajuda a enriquecer (Maley \& Bolitho, 2015) para posteriormente através da originalidade, ser criado ou produzido essa novidade (Clarke \& Lunt, 2014). A originalidade é constituída por propriedades observáveis publicamente (objetos físicos, estilos e comportamentos) enquanto a criatividade, em contraste, não é normalmente observável, mas imaginável (Brand, 2015). Finalmente, quando surge a definição de originalidade, ela é apresentada de diversos pontos de vista, tendo como base o tema de cada artigo e as conveniências do autor (Lancaster \& Culwin, 2007), (Glück, Ernst, \& Unger, 2010), (Kamp, Admiraal, \& Rijlaarsdam, 2016). Embora alguns autores tenham realizado um trabalho esforçado e de grande qualidade para explicar o que é a originalidade, verificou-se por análise desses artigos que o resultado desses trabalhos são muito particulares (Cremin, 1998), (Turcotte, Drolet, \& Girard, 2004), (Pavoine, Ollier, \& Dufour, 2005). Assim, pensamos que uma forma para resolver este problema é encontrar um conjunto de características pertencentes ao termo originalidade, que irá permitir identificar a sua existência ou falta dela em trabalhos científicos e/ou ajudar os interessados a construir uma definição correta e ampla do termo originalidade. Após esta identificação, apresentamos qual a diferença entre originalidade num artigo cientifico e numa tese de doutoramento.

\section{Objetivo da investigação}

O objetivo deste estudo é encontrar e apresentar um conjunto de características associados ao termo originalidade, que possa ser utilizado como guia em diversas áreas científicas, nomeadamente para identificar a 
originalidade em artigos científicos e em teses de doutoramento. Uma vez que ainda permanece a falta de precisão para aceitar de forma generalizada uma definição completa sobre originalidade, este estudo procura ajudar todos os intervenientes interessados (nomeadamente professores, alunos e membros de jornais científicos) a identificar "originalidade" de uma forma fácil, rigorosa e abrangente em artigos científicos ou teses doutoramento e/ ou ajudar todos aqueles que pretender construir uma definição sobre originalidade. Não é o objetivo desta investigação construir uma definição sobre originalidade.

\section{Fase 1 - Identificação das características}

\section{Os participantes}

Através da classificação de jornais científicos do SCImago para a categoria de "Educação", obtivemos uma lista ordenada de Jornais. Dessa lista foram selecionados os 100 melhores classificados. Posteriormente identificamos os membros correspondentes a esses jornais. Todos aqueles de que foi possível identificar o correio eletrônico foram selecionados para o questionário de pesquisa. No total trinta e três participantes completaram o questionário de pesquisa, identificados de acordo com a sua função em dois grandes grupos "Painel de revisão editorial" e "Editor chefe / Executivo / coeditor", ver figura 1.

FIGURA 1 - FUNÇÕES DOS PARTICIPANTES NO INQUÉRITO - FASE 1.

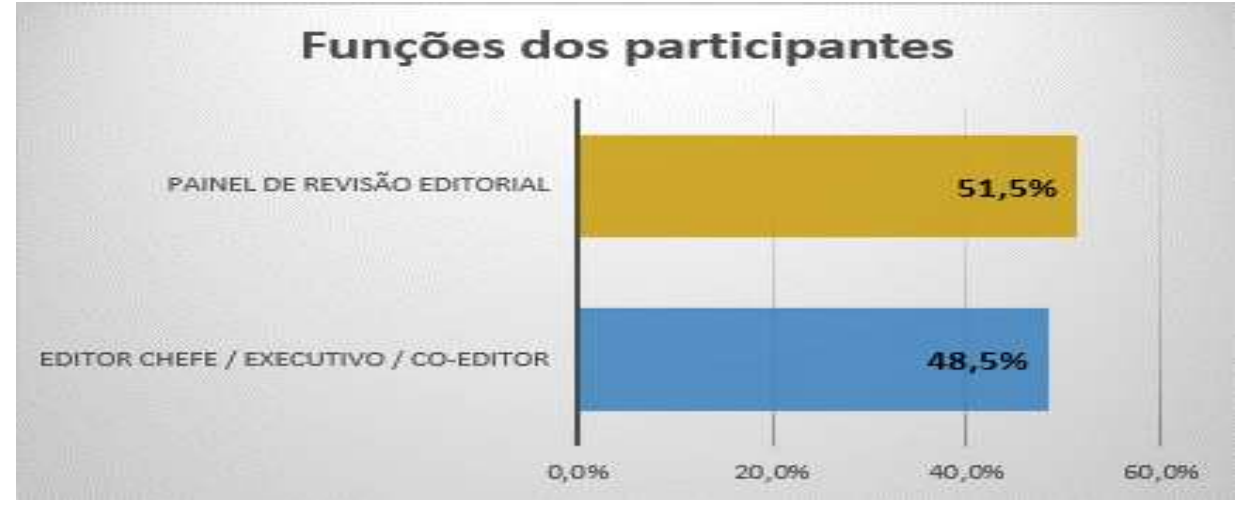

FONTE: Elaborado pelo autor. 


\section{O instrumento}

Foi criado um questionário, que era composto por cinco questões. As duas questões iniciais eram de resposta fechada (Draaijer, Hartog, \& Hofstee, 2007) e as outras três questões eram de resposta aberta (Powers, 2010). As perguntas abertas permitiam que os participantes escrevessem respostas completas usando o seu conhecimento / experiência, podendo escrever respostas curtas ou longas (Smyth, Dillman, Christian, \& Mcbride, 2009). O questionário começou pedindo aos participantes que se identificassem de acordo com a função que exercem no jornal cientifico. De seguida, perguntámos aos participantes se existia alguma diferença entre identificar a originalidade num artigo cientifico a ser publicado num jornal e identifica-la numa tese doutoramento. As opções de resposta eram "o mesmo" ou "diferente", e no caso da opção de resposta ser "diferente" então os participantes eram reencaminhados para uma pergunta aberta para que explicassem as diferenças existentes. Na terceira pergunta, pedíamos aos participantes para definirem originalidade de uma forma geral. A quarta questão pretendia saber quais eram as características para identificar originalidade num artigo cientifico. A quinta pergunta solicitava aos inquiridos que escrevessem as características que identifiquem a originalidade numa tese de doutoramento.

\section{Recolha de dados}

Os participantes foram convidados por correio eletrônico para participar nesta pesquisa, no e-mail enviado era explicado em detalhes que o questionário de pesquisa tinha como principal objetivo identificar as características de originalidade para um trabalho cientifico, em especial, num artigo e numa tese doutoramento, e perceber se existiam diferenças entre ambos. Amédia de tempo para completar a pesquisa foi de aproximadamente vinte e quatro minutos por participante. Após os inquéritos serem concluídos, os dados foram recolhidos, codificados e analisados.

\section{Análise dos dados}

As respostas obtidas através do questionário foram introduzidas numa folha do MS Excel. Posteriormente, foram distribuídas por tabelas e analisadas. Para a primeira e segunda pergunta foram utilizados métodos estatísticos simples, uma vez que eram questões fechadas. Para as restantes perguntas, ou seja, perguntas abertas, foi utilizado a metodologia qualitativa, inicialmente recorrendo a código aberto (Walker \& Myrick, 2006) e posteriormente ao código de correspondência (Kendall, 1999) para sintetizar, categorizar e 
relacionar temas. Doze características surgiram, ou seja, existe originalidade num trabalho cientifico se estivermos perante algo novo como: interpretação, metodologia, método, hipótese, tema, resultado, dados, testes, objetivos, pesquisa empírica, feito pelo autor e contribuição para o conhecimento. Várias estatísticas gerais foram realizadas em volta das perguntas do questionário e como a maioria dessas perguntas eram "abertas", a frequência total era superior ao número de inquiridos.

\section{Resultados da fase 1}

\section{O mesmo ou diferente}

Após os participantes se identificarem (pergunta 1, figura 1), o questionário prosseguia com a seguinte pergunta: "Identificar a originalidade num artigo cientifico é..." e as opções de resposta eram: a) o mesmo que identificar originalidade numa tese doutoramento; $b$ ) diferente do que identificar originalidade numa tese de doutoramento. A maioria dos participantes respondeu que era diferente $(67,9 \%)$, ver figura 2 .

FIGURA 2 - COMPARAÇÃO ENTRE TESE DE DOUTORAMENTO E ARTIGO CIENTIFICO.

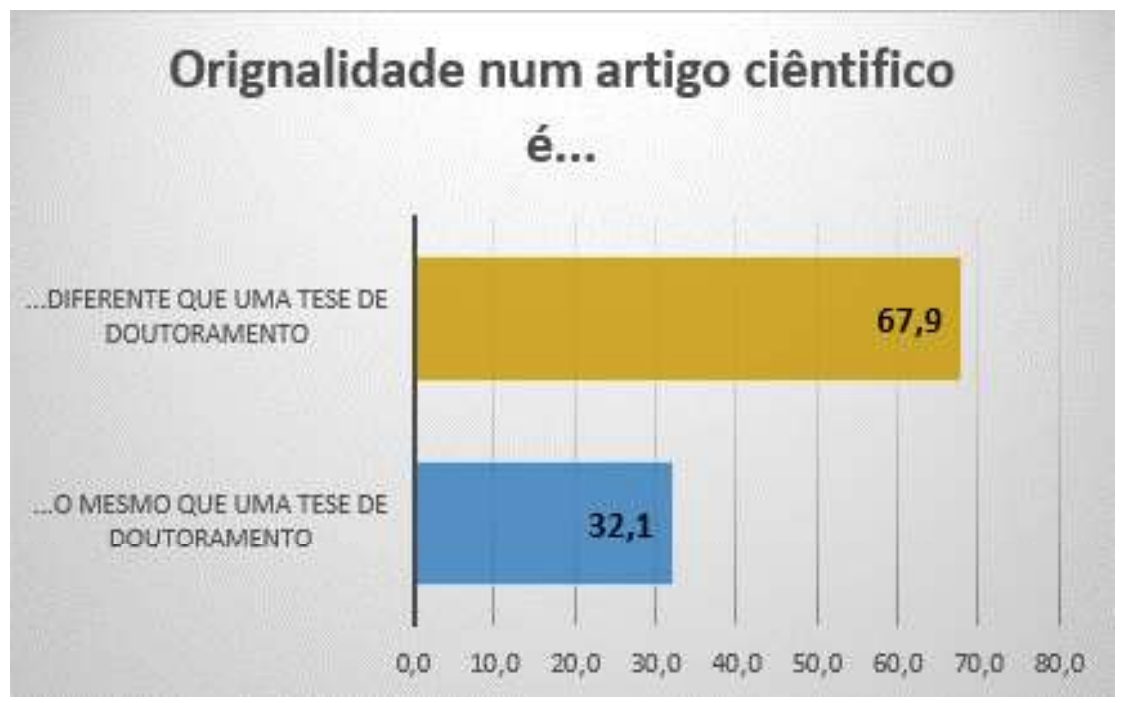

FONTE: Elaborado pelo autor. 


\section{Diferença existente}

Com base na pergunta anterior, para os participantes que respondiam "diferente do que identificar originalidade numa tese de doutoramento" o questionário convidava-os a explicar as diferenças através da questão 3 "É diferente por quê?". Dois itens surgiram como sendo as principais diferenças:

a) um artigo pode ser apenas qualitativo, uma tese doutoramento tem de mostrar capacidade para o inquérito;

b) um artigo pode ser escrito por um conjunto de autores, a tese de doutoramento deve ser o trabalho de um estudante.

\section{Como pode ser definido originalidade}

Na pergunta seguinte queríamos saber, de forma generalizada, como os inquiridos definem originalidade ("Como define originalidade em termos gerais?"). Com esta questão pretendíamos construir as características que identificassem a originalidade. Desta recolha de dados, doze características foram identificadas, ou seja, de uma forma geral, existe originalidade se, em comparação com outros trabalhos científicos já realizados anteriormente, estivermos na presença de: uma nova interpretação, uma nova metodologia, um novo método a ser utilizado, uma nova hipótese a ser utilizada, um novo tema, um novo resultado obtido, novos dados, novos testes a serem realizados, novos objetivos a serem alcançados, nova pesquisa empírica realizada, um trabalho realizado pelo autor e uma nova contribuição para o conhecimento cientifico.

\section{Características da originalidade num artigo submetido a um jornal}

Após conhecermos a opinião dos inquiridos sobre a definição de originalidade em termos gerais, foi o momento ideal para perguntar objetivamente quais são as características que identificam originalidade num artigo cientifico submetido a um jornal cientifico. Onze características foram identificadas pelos participantes, essas características devem conter algo de novo em relação a trabalhos anteriores, nomeadamente: interpretação, metodologia, método, hipótese, resultado, dados, testes, objetivos, pesquisa empírica, autoria, contribuição para o conhecimento cientifico.

\section{Características da originalidade para uma tese doutoramento}

Com o objetivo de conhecer o outro componente do título deste trabalho, isto é, identificar as características da originalidade numa tese doutoramento, 
foi utilizada a seguinte questão: "Quais são as características que identificam originalidade numa tese de doutoramento?". Doze características foram identificadas pelos participantes, ou seja, uma tese de doutoramento deve possuir algo de novo comparada com trabalhos de investigação anteriores (no caso de existirem) nomeadamente: interpretação, metodologia, método, hipótese, tema, resultado, dados, testes, objetivos, pesquisa empírica, autoria, contribuição para o conhecimento cientifico, ver figura 3.

Com base nas respostas das questões número 3, 4 e 5, foi construído um resumo apresentado na figura 3 que mostra as caraterísticas de originalidade em termos gerais e as diferenças entre as características da originalidade num artigo cientifico e as características de originalidade numa tese de doutoramento. Em termos do número de caraterísticas, a diferença está no tema, que deve ser novo numa tese de doutoramento, mas pode não ser novo num artigo cientifico.

FIGURA 3 - RESUMO DAS CARACTERÍSTICAS DE ORIGINALIDADE OBTIDAS PARA CADA PERGUNTA DO QUESTIONÁRIO, ELABORADO PELO AUTOR.

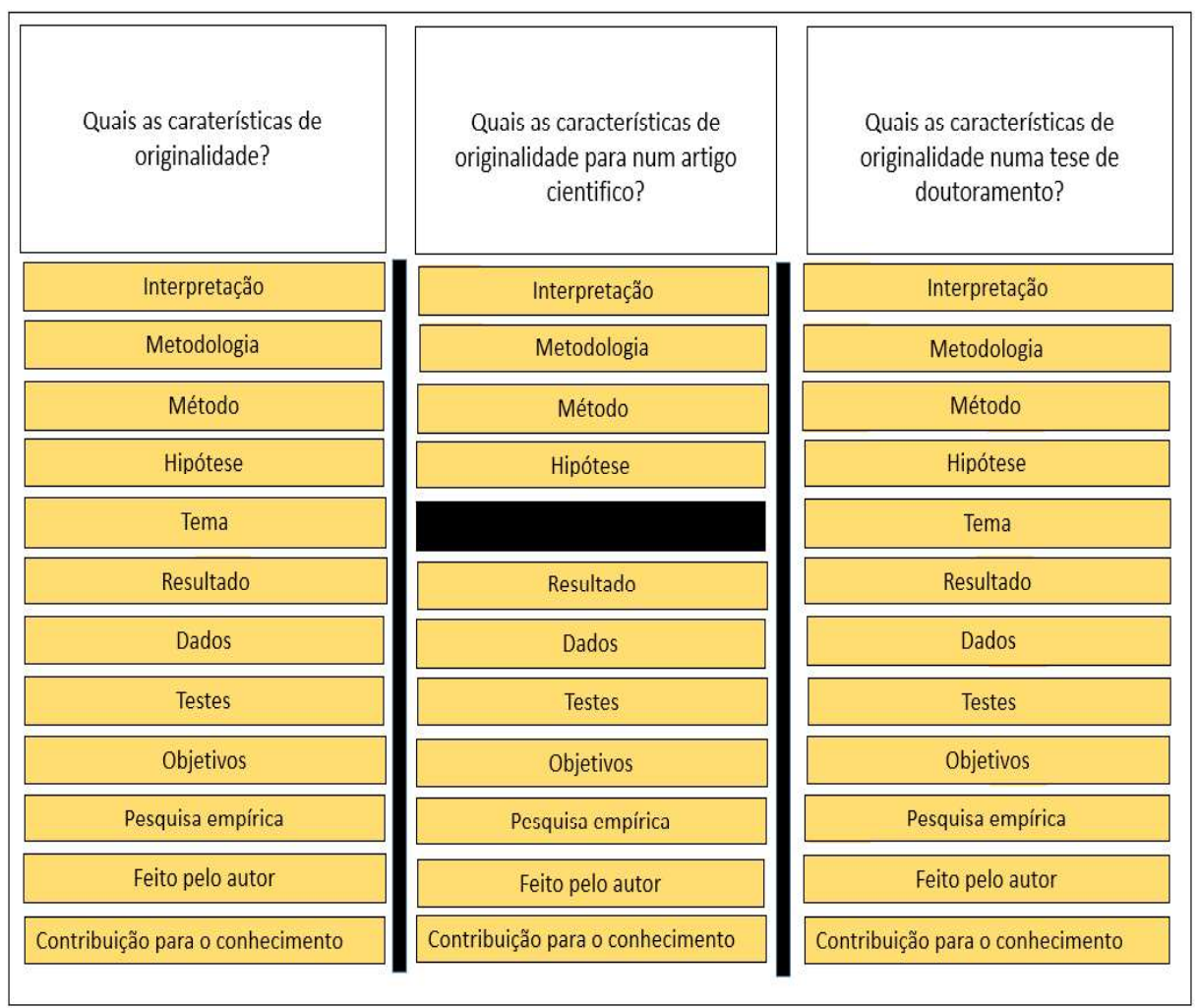

FONTE: Elaborado pelo autor. 


\section{Fase 2 - Validação das características}

\section{Os participantes}

Os mesmos participantes da fase 1, isto é, todos membros dos jornais que estavam nessa lista, foram igualmente convidados para o segundo questionário de pesquisa. No total quarenta e quatro participantes completaram o questionário de pesquisa, identificados de acordo com a sua função em dois grandes grupos "Painel de revisão editorial" e "Editor chefe / Executivo / coeditor", ver figura 4.

FIGURA 4 - FUNÇÕES DOS PARTICIPANTES NO INQUÉRITO - FASE 3.

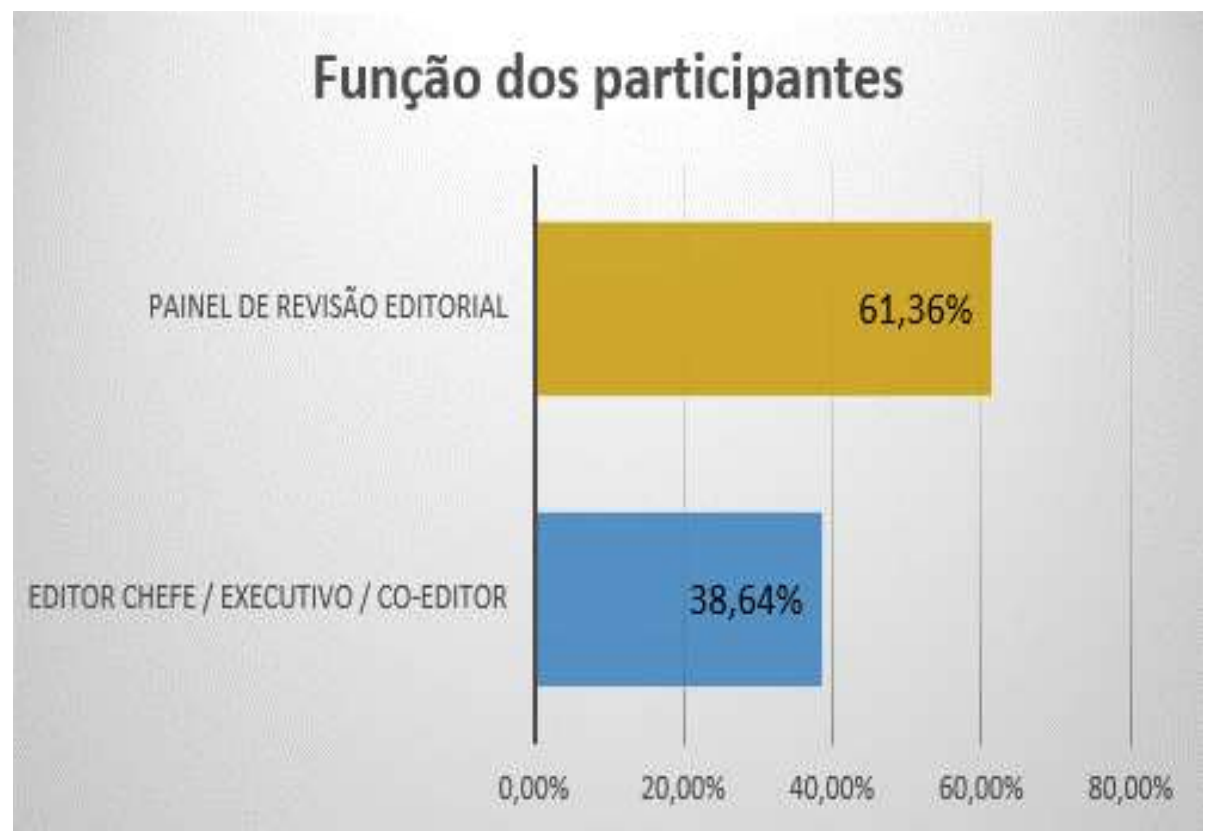

FONTE: Elaborado pelo autor.

\section{O instrumento}

Este questionário era composto por três questões, todas as questões eram de resposta fechada (Draaijer, Hartog, \& Hofstee, 2007). O questionário explicava aos participantes que o objetivo era validar as características encontradas num questionário anterior, o qual eles também tinham sido convidados a preencher. O questionário começou por pedir aos participantes que se identificassem de acordo com a função que exercem no jornal cientifico. Em seguida, perguntá- 
vamos aos participantes qual o seu grau de concordância ou discordância com as características apresentadas, que tinham sido construídas com base no questionário anterior. As opções de resposta para cada característica eram "discordo fortemente", "discordo", "discordo um pouco", "concordo um pouco, "concordo" e "concordo fortemente". Na terceira pergunta, pedíamos igualmente aos participantes para manifestassem o seu grau de concordância ou discordância relativamente à diferença entre originalidade numa tese de doutoramento e originalidade num artigo cientifico. As duas diferenças identificadas na fase 1 eram colocadas na mesma escala construída na pergunta anterior.

\section{Recolha de dados}

Os participantes foram convidados por correio eletrônico para participar nesta pesquisa, no correio eletrônico enviado era explicado com detalhe, que o questionário de pesquisa tinha como principal objetivo validar as características de originalidade para um artigo cientifico e para uma tese de doutoramento, características essas que foram identificadas num questionário anterior designada por fase 1. A média de tempo para completar a pesquisa foi de aproximadamente onze minutos por participante. Após os inquéritos serem concluídos, os dados foram recolhidos e analisados estatisticamente.

\section{Análise dos dados}

Todas as respostas obtidas através do questionário foram introduzidas numa folha do MS Excel em tabelas e analisadas com métodos estatísticos simples.

\section{Resultados da validação}

\section{Validação das caraterísticas}

Após os participantes se identificarem (pergunta 1, figura 4), a pergunta seguinte do questionário era: "Originalidade está associada a um conjunto de caraterísticas apresentadas em seguida, mencione o seu grau de concordância ou discordância para cada uma delas." A tabela 1 mostra todos os resultados obtidos. Ao observarmos os resultados da tabela 1, principalmente as três primeiras colunas da escala "Discordo fortemente", "Discordo" e "Discordo um pouco", podemos verificar que todas as caraterísticas são aceites, uma vez que estas três 
colunas apresentam pouca expressão de resultados em comparação com as três colunas seguintes, ou seja, o somatório de resultados das três primeiras colunas da escala ("Discordo fortemente", "Discordo" e "Discordo um pouco") totaliza 97 enquanto que o somatório das três colunas seguintes ("Concordo um pouco", "Concordo" e "Concordo fortemente") totaliza 431, a mesma lógica pode ser aplicada a cada característica, isto é, o somatório das três primeiras colunas da escala é sempre inferior ao somatório das três colunas seguintes.

TABELA 1: RESULTADO DA FREQUÊNCIA DE RESPOSTAS OBTIDO PARA A VALIDAÇÃO DAS CARATERÍSTICAS DE ORIGINALIDADE.

\begin{tabular}{lcccccc}
\hline \multicolumn{1}{c}{ Característica / Grau } & $\begin{array}{c}\text { Discordo } \\
\text { fortemente }\end{array}$ & Discordo & $\begin{array}{c}\text { Discordo } \\
\text { um } \\
\text { pouco }\end{array}$ & $\begin{array}{c}\text { Concordo } \\
\text { um pouco }\end{array}$ & Concordo & $\begin{array}{c}\text { Concordo } \\
\text { fortemente }\end{array}$ \\
\hline $\begin{array}{l}\text { Nova investigação } \\
\text { empírica }\end{array}$ & 6 & 8 & 6 & 6 & 10 & 8 \\
\hline Nova metodologia & 0 & 4 & 7 & 17 & 8 & 8 \\
\hline Novo método & 0 & 2 & 10 & 16 & 12 & 4 \\
\hline Novos testes & 0 & 4 & 4 & 18 & 14 & 4 \\
\hline Novo tema & 0 & 2 & 10 & 14 & 10 & 8 \\
\hline Novos objetivos & 0 & 4 & 2 & 16 & 20 & 2 \\
\hline Novos dados & 0 & 4 & 6 & 8 & 18 & 8 \\
\hline Feito pelo autor & 0 & 0 & 10 & 10 & 8 & 16 \\
\hline Nova hipótese & 0 & 0 & 4 & 12 & 20 & 8 \\
\hline Novo resultado & 0 & 0 & 4 & 12 & 10 & 18 \\
\hline Nova interpretação & 0 & 0 & 0 & 10 & 24 & 10 \\
\hline contribuição para & 0 & 0 & 0 & 0 & 10 & 34 \\
\hline
\end{tabular}

FONTE: Elaborado pelo autor. 
Por outro lado, se na tabela 1 introduzirmos uma nova linha designada por "Peso" (linha 1 da tabela 2) e atribuirmos um peso a cada coluna (grau de importância), em que, à coluna "Discordo fortemente" é atribuído um peso de 1 e à coluna "Concordo fortemente" é atribuído um peso de 6, e de seguida aplicar a fórmula em baixo para cada célula (pertencente a cada caraterística), obtemos um novo valor para cada coluna e consequentemente obtemos um total de grau de importância (ver tabela 2).

$$
=\sum(\mathrm{A} \times 1)+(\mathrm{B} \times 2)+(\mathrm{C} \times 3)+(\mathrm{D} \times 4)+(\mathrm{E} \times 5)+(\mathrm{F} \times 6)
$$

NOTA: Em que A corresponde à coluna "Discordo fortemente", B corresponde a "Discordo", C corresponde a "Discordo um pouco", D corresponde a "Concordo um pouco", E corresponde a "Concordo" e F corresponde a "Concordo fortemente".

FIGURA 5 - GRAU DE IMPORTÂNCIA DE CADA CARATERÍSTICA.

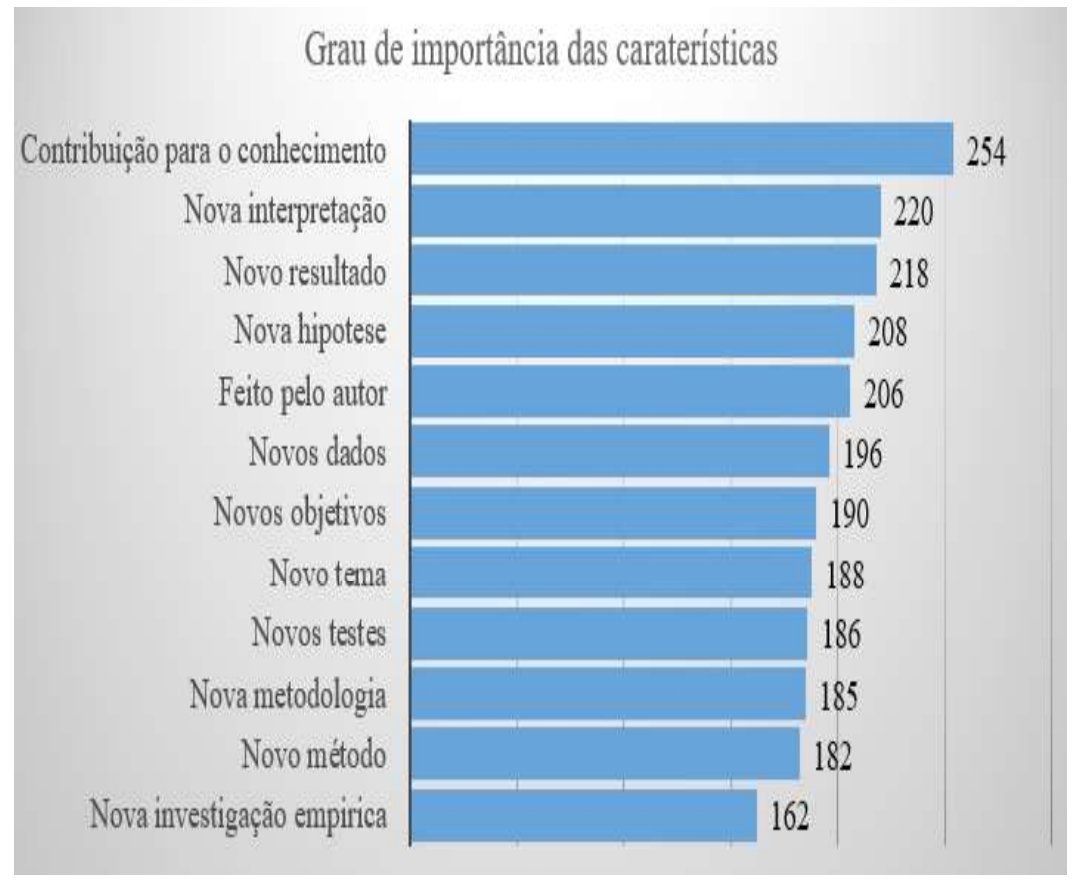

FONTE: Elaborado pelo autor. 
TABELA 2: INTRODUÇÃO DE "PESOS” PARA CADA GRAU DE SATISFAÇÃO DE CADA CARACTERÍSTICA.

\begin{tabular}{lccccccc}
\hline \multicolumn{1}{c}{ Peso $\rightarrow$} & 1 & 2 & 3 & 4 & 5 & 6 & \\
\hline $\begin{array}{c}\text { Característica } \\
\text { / Grau }\end{array}$ & $\begin{array}{c}\text { Discordo } \\
\text { fortemente }\end{array}$ & Discordo & $\begin{array}{c}\text { Discordo } \\
\text { um } \\
\text { pouco }\end{array}$ & $\begin{array}{c}\text { Concordo } \\
\text { um pouco }\end{array}$ & Concordo & $\begin{array}{c}\text { Concordo } \\
\text { fortemente }\end{array}$ & Total \\
\hline $\begin{array}{l}\mathrm{N} \text { o v a } \\
\text { investigação } \\
\text { empírica }\end{array}$ & 6 & 16 & 18 & 24 & 50 & 48 & 162 \\
\hline $\begin{array}{l}\text { N o v a } \\
\text { metodologia }\end{array}$ & 0 & 4 & 30 & 64 & 60 & 24 & 185 \\
\hline Novo método & 0 & 8 & 21 & 68 & 40 & 48 & 182 \\
\hline Novos testes & 0 & 8 & 12 & 72 & 70 & 24 & 186 \\
\hline Novo tema & 0 & 4 & 30 & 56 & 50 & 48 & 188 \\
\hline $\begin{array}{l}\text { N o v o s } \\
\text { objetivos }\end{array}$ & 0 & 8 & 6 & 64 & 100 & 12 & 190 \\
\hline Novos dados & 0 & 8 & 18 & 32 & 90 & 48 & 196 \\
\hline $\begin{array}{l}\text { Feito pelo } \\
\text { autor }\end{array}$ & 0 & 0 & 30 & 40 & 40 & 96 & 206 \\
\hline Nova hipótese & 0 & 0 & 12 & 48 & 100 & 48 & 208 \\
\hline $\begin{array}{l}\text { N o v o } \\
\text { resultado }\end{array}$ & 0 & 0 & 12 & 48 & 50 & 108 & 218 \\
\hline $\begin{array}{l}\text { N o v a } \\
\text { interpretação }\end{array}$ & 0 & 0 & 0 & 40 & 120 & 60 & 220 \\
\hline $\begin{array}{l}\text { Contribuição } \\
\text { para } \\
\text { conhecimento }\end{array}$ & 0 & 0 & 0 & 0 & 50 & 204 & 254 \\
\hline
\end{tabular}

FONTE: Elaborado pelo autor.

O total obtido na tabela 2 permite conhecer a importância de cada caraterística, ou seja, das doze as características identificadas a mais importante é "contribuição para o conhecimento" e a menos importante é "nova investigação empírica", no entanto todas elas são essenciais para identificar a originalidade nos trabalhos científicos. A figura 5 mostra o grau de importância de cada caraterística. 


\section{Validação das diferenças}

A validação das duas diferenças encontradas na questão 3 fase 1 era a última pergunta colocada neste questionário de avaliação, a mesma era a seguinte: "Com base nas características identificadas na pergunta anterior, a identificação de originalidade de um artigo em comparação a uma tese de doutoramento difere nos itens em baixo. Por favor, indique o grau de concordância ou discordância para cada item.". A escala de resposta era igual à pergunta anterior, ou seja, "Discordo fortemente", "Discordo", "Discordo um pouco", "Concordo um pouco", "Concordo" e "Concordo fortemente". A tabela 3 mostra a frequência de todas as respostas. À semelhança da análise realizada para validar as características, observamos que as diferenças foram aceites, uma vez que, as três colunas da escala designadas por "Discordo fortemente", "Discordo" e "Discordo um pouco" apresentam pouca expressão de resultados em comparação com as três colunas seguintes, ou seja, o somatório dos resultados das três primeiras colunas da escala ("Discordo fortemente", "Discordo" e "Discordo um pouco") totaliza 30 enquanto que o somatório das três colunas seguintes ("Concordo um pouco", "Concordo" e "Concordo fortemente") totaliza 144.

TABELA 3: RESULTADO DA FREQUÊNCIA DE RESPOSTAS OBTIDO PARA A VALIDAÇÃO DAS DIFERENÇAS DE ORIGINALIDADE DE UM ARTIGO CIENTÍFICO E DE UMA TESE DE DOUTORAMENTO.

\begin{tabular}{lcccccc}
\hline \multicolumn{1}{c}{ Diferença / Grau } & $\begin{array}{c}\text { Discordo } \\
\text { fortemente }\end{array}$ & Discordo & $\begin{array}{c}\text { Discordo } \\
\text { um } \\
\text { pouco }\end{array}$ & $\begin{array}{c}\text { Concordo } \\
\text { um pouco }\end{array}$ & Concordo & $\begin{array}{c}\text { Concordo } \\
\text { fortemente }\end{array}$ \\
\hline $\begin{array}{l}\text { Artigo - Pode ser } \\
\text { apenas qualitativo }\end{array}$ & 4 & 4 & 8 & 12 & 8 & 6 \\
\hline $\begin{array}{l}\text { Artigo - Um ou vários } \\
\text { autores }\end{array}$ & 2 & 0 & 4 & 8 & 10 & 20 \\
$\begin{array}{l}\text { Tese doutoramento } \\
- \text { Um único autor (o }\end{array}$ & 2 & 0 & 4 & 0 & 18 & 20 \\
estudante) \\
$\begin{array}{l}\text { Tese doutoramento } \\
-\quad \text { Deve mostrar } \\
\text { capacidade para o } \\
\text { inquérito }\end{array}$
\end{tabular}

FONTE: Elaborado pelo autor. 
Com a atribuição do "Peso" (linha 1 da tabela 4) em cada coluna (grau de importância), em que à coluna "Discordo fortemente" é atribuído um peso de $1 \mathrm{e}$ à coluna "Concordo fortemente" é atribuído um peso de 6, e com a aplicação de uma formula (em baixo) para cada célula (pertencente a cada diferença), obtemos um novo valor para cada coluna e consequentemente um total (ver tabela 4).

$$
=\sum(\mathrm{A} \times 1)+(\mathrm{B} \times 2)+(\mathrm{C} \times 3)+(\mathrm{D} \times 4)+(\mathrm{E} \times 5)+(\mathrm{F} \times 6)
$$

NOTA: Em que A corresponde a "Discordo fortemente", B corresponde a "Discordo", C corresponde a "Discordo um pouco", D corresponde a "Concordo um pouco", E corresponde a "Concordo" e F corresponde a "Concordo fortemente".

TABELA 4: INTRODUÇÃO DE "PESOS” PARA CADA GRAU DE SATISFAÇÃO DE CADA CARACTERÍSTICA.

\begin{tabular}{|c|c|c|c|c|c|c|c|}
\hline $\begin{array}{c}\text { Diferença / } \\
\text { Grau }\end{array}$ & $\begin{array}{l}\text { Discordo } \\
\text { fortemente }\end{array}$ & Discordo & $\begin{array}{l}\text { Discordo } \\
\text { um } \\
\text { pouco }\end{array}$ & $\begin{array}{l}\text { Concordo } \\
\text { um pouco }\end{array}$ & Concordo & $\begin{array}{l}\text { Concordo } \\
\text { fortemente }\end{array}$ & Total \\
\hline $\begin{array}{l}\text { Artigo - Pode } \\
\text { ser apenas } \\
\text { qualitativo }\end{array}$ & 4 & 8 & 24 & 48 & 40 & 36 & 700 \\
\hline $\begin{array}{l}\text { Artigo - Um ou } \\
\text { vários autores }\end{array}$ & 2 & 0 & 12 & 32 & 50 & 120 & 1136 \\
\hline $\begin{array}{l}\mathrm{T} \text { e } \mathrm{s} \quad \mathrm{e} \\
\text { doutoramento }- \\
\text { Um único autor } \\
\text { (o estudante) }\end{array}$ & 2 & 0 & 12 & 0 & 90 & 120 & 1208 \\
\hline $\begin{array}{l}\mathrm{T} \text { e } \mathrm{s} \text { e } \\
\text { doutoramento } \\
\text { - Deve mostrar } \\
\text { capacidade para } \\
\text { o inquérito }\end{array}$ & 0 & 0 & 6 & 24 & 70 & 132 & 1256 \\
\hline
\end{tabular}

FONTE: Elaborado pelo autor. 


\section{Fase 3 - Exemplos de descrição para as características}

Após a identificação e validação das caraterísticas de originalidade, entendemos que devíamos complementar este trabalho com exemplos de descrição das características descobertas. Estes exemplos, pretendem auxiliar-nos a identificar mais facilmente e rapidamente a originalidade em trabalhos científicos. Para nos ajudar a construir os exemplos de descrição para cada característica, selecionamos 48 artigos que tinham relevância para este trabalho, eles estavam distribuídos em vários repositórios científicos internacionais. Relevância é um conceito central na ciência da informação devido à sua importância para a pesquisa no campo da documentação, informação e/ou informação cientifica (Bookstein, 1979), (Hersh, 1994) e (Kotkas, Holbrook, \& Rannikmäe, 2016), ou seja, o resultado da pesquisa sobre o assunto a investigar possui pelo menos um componente importante de informação contida nos documentos consultados e que permite responder ou ajudar à investigação em curso (Barry, 1994) e (Muddiman \& Frymier, 2009).

\section{Base de dados utilizada}

A estratégia de selecionar vários repositórios de informação para a revisão da literatura deve-se ao facto de garantir o máximo de qualidade e rigor para este trabalho. Neste contexto, selecionamos os trabalhos científicos relevantes dos repositórios que possuem impacto significativo na ciência devido à sua grande reputação na publicação de artigos e consulta para novos trabalhos científicos ou melhoramento destes: EBSO (Shapiro, 2013), ERIC (Jacsó, 2007), SAGE e Scopus (Bar-Ilan, 2007).

\section{Revisão da literatura}

A originalidade é um aspeto intrínseco do valor de todo o trabalho de investigação (Golden-Biddle \& Locke, 2007). Originalidade é usualmente entendida como alguma coisa nova, que não foi feita anteriormente (Lancaster $\&$ Culwin, 2007), (Moore, 2007), em que unimos diversos elementos para criar 
um todo, novo, coerente e que resultará no original escrito pela primeira vez (Krathwohl, 2002). A originalidade pode ser identificada de diversas formas (Rhoten, O'Connor, \& Hackett, 2009), uma forma muito básica e simples de a identificar é nos perguntarmos: a) o que difere o trabalho científico que está a ser feito de outros trabalhos semelhantes realizados anteriormente? (no caso de existirem trabalhos semelhantes), isto é, o que há de novo? (Holbrook, Bourke, Lovat, \& Dally, 2005), b) qual o valor que adiciona à ciência? (Webster \& Watson, 2002), e c) para que serve o meu trabalho (objetivo, propósito, contribuição para o conhecimento)? (Mu, 2015), (Ortinau, 2011). Um modelo muito mais "sofisticado" que o anterior mas pouco detalhado foi construído por Mårtenssona et al. (2016), que identifica a originalidade de um trabalho científico tendo em conta a ideia, o procedimento e o resultado; ou seja, segundo os autores se estes itens são novos então existe originalidade. Coniam (2012) também identifica três itens, embora diferentes dos autores anteriores: contribuição para o conhecimento, aceitabilidade das reivindicações e investigação de acordo com o âmbito. Para além dos artigos acima consultados, outro conjunto de artigos permitiu concluir que nós podemos identificar a existência de originalidade num artigo cientifico ou numa tese de doutoramento recorrendo a grupo de nove perguntas: I) O tipo de investigação científica utilizado para a investigação é diferente do que foi feito anteriormente? (Neuman, 2014), por exemplo é uma investigação estatística, comparativa, experimental, histórica, quase-experimental, casual, comparativa, correlação, de diferente interpretação, um estudo de caso, um estudo de campo, etnográfico, bibliográfico, empírico, investigação-ação (Boyle, et al., 2014), (Currie, 2016), (Rakap, 2015), (Horsford \& D'Amico, 2015); II) o método cientifico utilizado é diferente daquele utilizado anteriormente? (Ifenthaler \& Seel, 2013), por exemplo foi utilizado o método dedutivo, indutivo ou hipotético-dedutivo (Sik, 2015); III) a metodologia utilizada na pesquisa atual é diferente da anterior? (Nye, Melendez-Torres, \& Bonell, 2016), por exemplo foi empregue a metodologia qualitativa, quantitativa ou ambas (Eyisi, 2016); IV) os instrumentos de recolha de dados são diferentes comparado com o trabalho anterior? (Spence, Lachlan, \& Rainear, 2016), isto é, os questionários realizados (Hong \& Choi, 2015), as entrevistas realizadas (Roulston, 2014), a análise estatística realizada, que deve apresentar interpretações diferentes (Aminghafari, Cheze, \& Poggi, 2006), a análise de conteúdo realizada deve ser diferente (Faucher \& Garner, 2015), ou as checklists utilizadas devem ser diferentes (Tosh, 2015), (Owen \& Baum, 1985); V) as técnicas de recolha de dados utilizadas são diferentes? (Robinson, Emden, Croft, \& Vosper, 2011) e (Johnson \& Clarke, 2003), isto é, a observação (Webster R. , 2015), a análise documental (O’Donnell, 2016), o inquérito (Ting \& Ho, 2015), os testes de validação que foram realizados são diferentes ou nunca foram reali- 
zados (Haertel, 2013), o grupo de focos é diferente (Faucher \& Garner, 2015), o portfólio do investigador é diferente (Hack, 2015), (Zhong \& Jiang, 2016) ou são utilizadas outras formas como por exemplo o método delphi (Geist, 2010), (Gill, Leslie, Grech, \& Latour, 2013); VI) as hipóteses são diferentes, isto é, as combinações possíveis de acordo com a operação realizada com as variáveis de investigação são diferentes? (Stiburek, 2016), por exemplo é utilizado uma variável dependente, independente, controle, interveniente (Meneses, Fàbregues, Rodríguez-Gómez, \& Ion, 2012); VII) o tema é diferente, isto é, o contexto, o tópico (Soler, 2011) ou o ponto de partida do tema? (Dernt, 2014); VIII) os resultados são diferentes, isto é, a descrição dos relatos encontrados é diferente? (Heitzmann, 2008), como por exemplo apresentá-los em forma de texto, dados, figuras, gráficos (Ahl, 2016) e (Houts, Doak, Doak, \& Loscalzo, 2006); IX) os objetivos traçados para a investigação são novos? (Ambrose \& Kulik, 1999), por exemplo, explorar outras direções (Runco, Illies, \& Eisenman, 2005), outras estratégias (Wood \& Tarrier, 2010), outra motivação ou criatividade (Smith \& Yang, 2004).

\section{Recolha e análise dos dados}

Durante a leitura e análise dos 48 artigos da revisão de literatura, foram introduzidas numa tabela no MS Excel todas as frases chave. Posteriormente recorreu-se a códigos abertos (Walker \& Myrick, 2006) e ao código de correspondência (Kendall, 1999) para sintetizar, categorizar e relacionar características emergentes. Agregado às doze características identificadas na fase 1 surgiram um conjunto de exemplos de descrição/significado: interpretação (estatística, comparativa, experimental, histórica), metodologia (qualitativa, quantitativa ou mista), método (dedutivo, indutivo e hipotético-dedutivo), hipótese (variável dependente, independente, controle, novos dados), tema (tópico, contexto, ponto de partida), resultado (descrição dos relatos encontrados - podem ser textos, dados, figuras, gráficos ou outras formas de apresentação), dados (instrumentos de recolha: questionários, entrevistas, análise estatística, análise de conteúdo + técnicas de recolha de dados: observação, análise documental, inquérito, testes de validação, grupo de focos, Delphi), testes (validação, complemento, outro método, outro procedimento), objetivos (explorar outras direções, outras estratégias, criatividade), pesquisa empírica, feito pelo autor, contribuição para o conhecimento (nova descoberta publicada que permite evoluir e desenvolver novas ideias). 


\section{Resultado}

As características encontradas e complementadas na revisão da literatura com um conjunto de exemplos de descrição/significado, resultou num esquema gráfico apresentado na figura 6 . Este esquema simples e claro permite identificar as características diretamente ligadas ao termo de originalidade e permite também visualizar alguns exemplos de descrição dessas características. Assim, a figura 6 deve ser interpretada desta forma:

- características de originalidade - constituído pelo primeiro anel imaginário ligado à caixa central e que é composto pelas características: interpretação, metodologia, método, hipótese, tema, resultado, dados, testes, objetivos, pesquisa empírica, feito pelo autor e contribuição para o conhecimento;

- exemplos de descrição - constituído pelo segundo anel imaginário ligado a cada característica.

FIGURA 6 - CARACTERÍSTICAS DE ORIGINALIDADE E EXEMPLOS DE DESCRIÇÃO, ELABORADO PELO AUTOR.

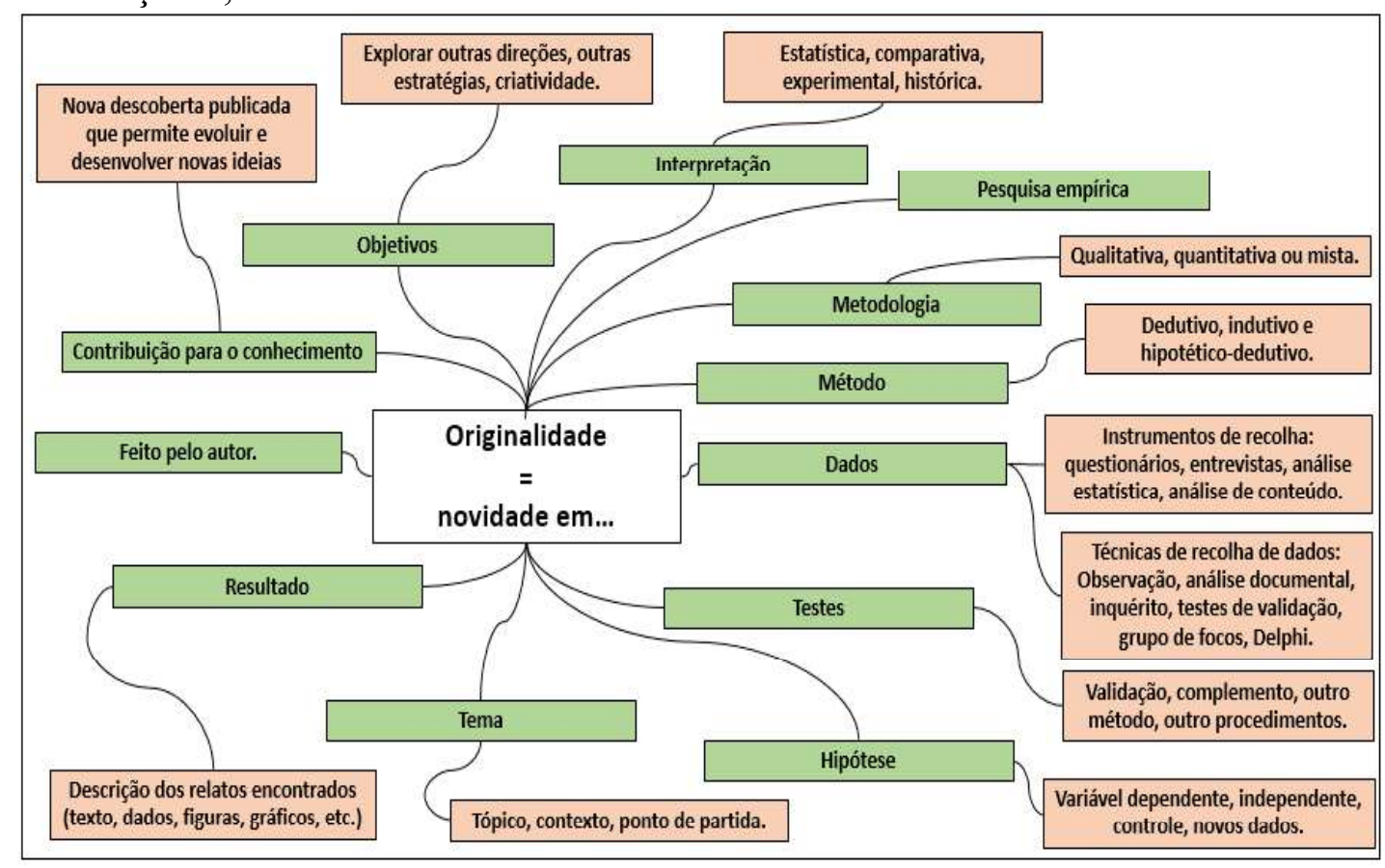

FONTE: Elaborado pelo autor. 
De forma semelhante, foi igualmente construído um esquema gráfico correspondente às diferenças entre a identificação de originalidade num artigo científico e numa tese de doutoramento. Como foi dito na primeira fase deste estudo, existem duas grandes diferenças: o número de autores que realizam o trabalho (um artigo pode ser escrito por um conjunto de autores, mas uma tese de doutoramento deve ser o trabalho apenas do estudante) e a metodologia (um artigo pode ser apenas qualitativo, mas uma tese doutoramento tem de mostrar capacidade para o inquérito). A figura 7 mostra as diferenças.

FIGURA 7 - DIFERENÇA ENTRE ORIGINALIDADE NUMA TESE DE DOUTORAMENTO E UM ARTIGO CIENTÍFICO, ELABORADO PELO AUTOR.

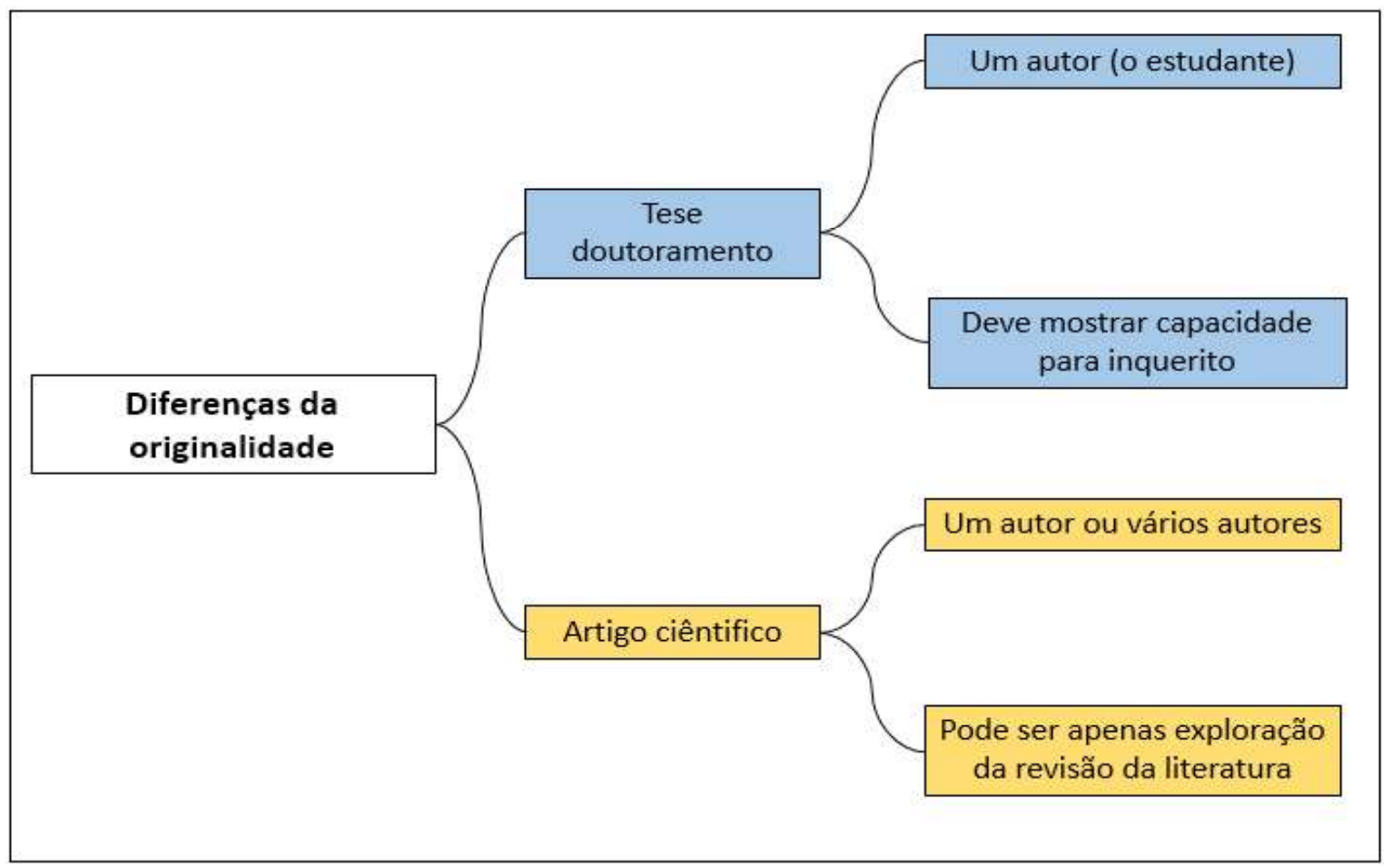

FONTE: Elaborado pelo autor.

\section{Conclusão}

$\mathrm{Na}$ investigação científica, a originalidade tem um papel importante, na medida em que, as descobertas originais são fundamentais para o progresso científico. Contudo, não foi encontrado por nós nenhum artigo científico que contivesse um conjunto de características para identificar facilmente a originalidade num trabalho científico. Neste contexto, o nosso trabalho permitiu 
encontrar um conjunto de características ligadas à originalidade e, em conjunto com a revisão de literatura, construímos uma pequena descrição ligada a cada caraterística. Uma representação em forma guia (figura 6 e 7) ajudará aos interessados a reconhecer a originalidade em trabalhos científicos, de uma forma simples e clara.

Esta pesquisa permite concluir que os trabalhos desenvolvidos anteriormente por outros autores centram-se em definições, muitas delas complexas, outras incompletas e outras centradas apenas nos trabalhos de pesquisa que desenvolvem, ou seja, pouco abrangentes e pouco generalizadas. Com recurso somente a esses trabalhos é difícil identificar a originalidade de um trabalho científico. Por esta razão, uma possível solução para eliminar estes pontos fracos, é utilizar as características encontradas neste trabalho e criar uma única forma de identificar originalidade. A representação da figura 6 e a figura 7 é abrangente, generalizado e universal para qualquer aluno, professor, membro de jornal ou outro interessado possa identificar a originalidade ou, alguém que pretenda construir uma definição.

Em suma, neste momento há uma base de trabalho uniforme. Dado a importância deste tema, mas também a sua complexidade, para trabalhos futuros, torna-se necessário explorar a hipótese de construção de uma ferramenta simples e prática que ajude todos os interessados a identificar automaticamente a originalidade num trabalho científico.

\section{REFERÊNCIAS}

AHL, L. M. (2016). Research Findings' Impact on the Representation of Proportional Reasoning in Swedish Mathematics Textbooks. Journal of Research in Mathematics Education, 5(2), 180-204.

AMBROSE, M. L. \& KULIK, C. T. (1999). Old Friends, New Faces: Motivation Research in the 1990s. Journal of Management, 25(3), 231-292.

AMINGHAFARI, M.; CHEZE, N.; \& POGGI, J.-M. (2006). Multivariate denoising using wavelets and principal component analysis. Computational Statistics \& Data Analysis, 50(9), 2381-2398.

BAR-ILAN, J. (2007). Which h-index? - A comparison of WoS, Scopus and Google Scholar. Scientometrics, 74(2), 257-271.

BARRY, C. L. (1994). User-defined relevance criteria: An exploratory study. Journal of the Association for Information Science and Technology, 3, 149-159. 
BOOKSTEIN, A. (1979). Relevance. Journal of the American Society for Information Science, 30(5), 269-273.

BOURKE, S.; \& HOLBROOK, A. P. (2011). Examining PhD and research masters theses. Assessment \& Evaluation in Higher Education., 38(4), 407-416.

BOYLE, E. A.; MACARTHUR, E. W.; CONNOLlY, T. M.; HAINEY, T.; MANEA, M.; KÄRCKI, A., \& ROSMALEN, P. v. (2014). A narrative literature review of games, animations and simulations to teach research methods and statistics. Computers \& Education, 74, 1-14.

BRAND, P. Z. (2015). The Role of Luck in Originality and Creativity. The Journal of Aesthetics and Art Criticism, 73(1), 31-55.

BROOKS, J. S.; \& NORMORE, A. H. (2015). Qualitative research and educational leadership: Essential dynamics to consider when designing and conducting studies. International Journal of Educational Management, 29(7), 798-806.

CLARKE, G.; \& LUNT, I. (2014). The concept of 'originality' in the Ph.D.: how is it interpreted by examiners? Assessment \& Evaluation in Higher Education, 39(7), 803-820.

CONIAM, D. (2012). Exploring reviewer reactions to manuscripts submitted to academic journals. System, 40(4), 544-553.

CREMIN, M. (1998). The Imagination, and Originality, in English and Classroom Drama. English in Education, 32(2), 4-13.

CROPLEY, A. J. (2000). Defining and measuring creativity: Are creativity tests worth using? Roeper Review, 23(2), 72-79.

CURRIE, A. (2016). Ethnographic analogy, the comparative method, and archaeological special pleading. Studies in History and Philosophy of Science Part A, 55, 84-94.

DERNT, M. (2014). Basics of research paper writing and publishing. International Journal of Technology Enhanced Learning, 6(2), 105-123.

DRAAIJER, S.; HARTOG, R. J.; \& HOFSTEE, J. (2007). Guidelines for the Design of Digital Closed Questions for Assessment and Learning in Higher Education. E-Journal of Instructional Science and Technology, 10(1), 1-29.

EYISI, D. (2016). The Usefulness of Qualitative and Quantitative Approaches and Methods in Researching Problem-Solving Ability in Science Education Curriculum. Journal of Education and Practice, 7(15), 91-100.

FAUCHER, M. A.; \& GARNER, S. L. (2015). A method comparison of photovoice and content analysis: research examining challenges and supports of family caregivers. Applied Nursing Research, 28(4), 262-267.

GEIST, M. R. (2010). Using the Delphi method to engage stakeholders: A comparison of two studies. Evaluation and Program Planning, 33(2), 147-154. 
GILL, F. J., LESLIE, G. D.; GRECH, C.; \& LATOUR, J. M. (2013). Using a web-based survey tool to undertake a Delphi study: Application for nurse education research. Nurse Education Today, 33(1), 1322-1328.

GLÜCK, J.; ERNST, R.; \& UNGER, F. (2010). How Creatives Define Creativity: Definitions Reflect Different Types of Creativity. Creativity Research Journal, 14(1), 55-67.

GOLDEN-BIDDLE, K.; \& LOCKE, K. (2007). Composing qualitative research. (Second Edition ed.). Sage Publications, Inc.

HACK, C. (2015). Analytical rubrics in higher education: A repository of empirical data. British Journal of Educational Technology, 5, 924-927.

HAERTEL, E. (2013). How Is Testing Supposed to Improve Schooling? Measurement: Interdisciplinary Research and Perspectives, 11(1-2), 1-18.

HARWOOD, N. (2005). 'Nowhere has anyone attempted ... In this article I aim to do just that': A corpus-based study of self-promotional I and we in academic writing across four disciplines. Journal of Pragmatics, 37(8), 1207-1231.

HEITZMANN, R. (2008). Writing for Publication in Social Studies Education. Social Studies, 99(1), 37-40.

HERSH, W. (1994). Relevance and retrieval evaluation: Perspectives from medicine. Journal of the American Society for Information Science, 45(3), 201-206.

HOLBROOK, A.; BOURKE, S.; LOVAT, T.; \& DALLY, K. (2005). Investigating PhD thesis examination reports. International Journal of Educational Research, 41(2), 98-120.

HONG, Y.-C.; \& CHOI, I. (2015). Assessing reflective thinking in solving design problems: The development of a questionnaire. British Journal of Educational Technology, 46(4), 848-863.

HORSFORD, S. D.; \& D'AMICO, D. (2015). The past as more than prologue: a call for historical research. International Journal of Educational Management, 29(7), 863-873.

HOUTS, P. S.; DOAK, C. C.; DOAK, L. G.; \& LOSCALZO, M. J. (2006). The role of pictures in improving health communication: A review of research on attention, comprehension, recall, and adherence. Patient Education and Counseling, 61(2), 173-19.

IFENTHALER, D.; \& SEEL, N. M. (2013). Model-based reasoning. Computers \& Education, 64, 131-142.

JACSÓ, P. (2007). How big is a database versus how is a database big. Online Information Review, 31(4), 533-536.

JOHNSON, B.; \& CLARKE, J. M. (2003). Collecting Sensitive Data: The Impact on Researchers. Qualitative Health Research, 13(3), 421-434.

JONASSEN, D. H. (2000). Toward a design theory of problem solving. Educational Technology Research and Development, 48(4), 63-85. 
KAMP, M.-T. V.; ADMIRAAL, W.; \& RIJLAARSDAM, G. (2016). Becoming Original: Effects of Strategy Instruction. Instructional Science: An International Journal of the Learning Sciences, 44(6), 543-566.

KENDALL, J. (1999). Axial Coding and the Grounded Theory Controversy. Western Journal of Nursing Research, 21(6), 743-757.

KOTKAS, T.; HOLBROOK, J.; \& RANNIKMÄE, M. (2016). Identifying Characteristics of Science Teaching/Learning Materials Promoting Students' Intrinsic Relevance. Science Education International, 27(2), 194-216.

KRATHWOHL, D. R. (2002). A Revision of Bloom's Taxonomy: An Overview. Theory Into Practice, 41(4), 212-218.

KYVIK, S.; \& THUNE, T. (2014). Assessing the quality of PhD dissertations. A survey of external committee members. Assessment \& Evaluation in Higher Education, 40(5), 768-782.

LANCASTER, T.; \& CULWIN, F. (2007). Preserving academic integrity—fighting against nonoriginality agencies. British Journal of Educational Technology, 38(1), 153-157.

LUNDGREN, S. M.; \& ROBERTSSON, B. (2013). Writing a bachelor thesis generates transferable knowledge and skills useable in nursing practice. Nurse education today, 33(11), 1406-1410.

MALEY, A.; \& BOLITHO, R. (2015). Creativity. ELT journal, 69(4), 434-436.

MARTENSSONA, P.; FORSB, U.; WALLINC, S.-B.; ZANDERD, U.; \& NILSSON, G. H. (2016). Evaluating research: A multidisciplinary approach to assessing research practice and quality. Research Policy, 45(3), 593-603.

MENESES, J.; FÀBREGUES, S.; RODRÍGUEZ-GÓMEZ, D.; \& ION, G. (2012). Internet in teachers' professional practice outside the classroom: Examining supportive and management uses in primary and secondary schools . Computers \& Education, 59(3), 915-924.

MOORE, B. (2007). Original sin and insider research. Action Research, 5(1), 27-39.

MU, C. (2015). Writing and publishing science research papers in english: A global perspective. English for Specific Purposes, 39, 76-78.

MUDDIMAN, A.; \& FRYMIER, A. B. (2009). What is Relevant? Student Perceptions of Relevance Strategies in College Classrooms. Communication Studies, 60(2), 130-146.

NEUMAN D. (2014). Qualitative research in educational communications and technology: a brief introduction to principles and procedures. Journal of Computing in Higher Education, 26(1), 69-86.

NYE, E., MELENDEZ-TORRES G. J.; \& BONELL, C. (2016). Origins, methods and advances in qualitative meta-synthesis. Review of Education, 4(1), 57-79. 
O’DONNELL, V. L. (2016). Organisational change and development towards inclusive higher education. Journal of Applied Research in Higher Education, 8(1), 101-119.

ORTINAU, D. J. (2011). Writing and publishing important scientific articles: A reviewer's perspective. Journal of Business Research, 64(2), 150-156.

OWEN, S. V.; \& BAUM S. M. (1985). The Validity of the Measurement of Originality. Educational and Psychological Measurement, 45(4), 939-944.

PAVOINE, S.; OLLIER, S.; \& DUFOUR, A.-B. (2005). Is the originality of a species measurable? Ecology Letters, 8(6), 579-586.

PENG, H. (2015). Assessing the quality of research supervision in mainland Chinese higher education. Quality in Higher Education, 21(1), 89-100.

PIETERS, R.; WARLOP, L.; \& WEDEL, M. (2002). Breaking Through the Clutter: Benefits of Advertisement Originality and Familiarity for Brand Attention and Memory. Management Science, 48(6), 765-781.

POWERS, Y. A. (2010). Immediate Feedback and Opportunity to Revise Answers to Open-Ended Questions. Educational and Psychological Measurement., 70(1).

RAKAP, S. (2015). Effect sizes as result interpretation aids in single-subject experimental research: description and application of four nonoverlap methods. British Journal of Special Education, 42(1), 11-33.

RHOTEN, D.; O'CONNOR, E.; \& HACKETT, E. J. (2009). The Act of Collaborative Creation and the Art of Integrative Creativity: Originality, Disciplinarity and Interdisciplinarity. Thesis Eleven, 96(1), 83-108.

ROBINSON, A. L.; EMDEN, C. G.; CROFT, T. D.; \& VOSPER, G. C. (2011). Mixed Methods Data Collection in Dementia Research. Journal of Mixed Methods Research, 5(4), 330-344.

ROULSTON, K. (2014). Interactional problems in research interviews. Qualitative Research, 14(3), 277-293.

RUNCO, M. A. (2015). Meta-Creativity: Being Creative About Creativity. Creativity Research Journal, 27(3), 295-298.

RUNCO, M. A.; ILLIES, J. J.; \& EISENMAN, R. (2005). Creativity, Originality, and Appropriateness: What do Explicit Instructions Tell Us About Their Relationships? The Journal of Creative Behavior, 39(2), 137-148.

SERENKO, A.; BONTIS, N.; BOOKER, L.; SADEDDIN, K.; \& HARDIE, T. (2010). A scientometric analysis of knowledge management and intellectual capital academic literature (1994-2008). Journal of Knowledge Management, 14(1), 3-23.

SHAPIRO, S. (2013). Are Aggregators an Endangered Species? Journal of Electronic Resources Librarianship, 25(1), 77-79. 
SIK, K. (2015). Tradition or Modernism in Grammar Teaching: Deductive vs. Inductive Approaches. Procedia - Social and Behavioral Sciences, 197, 2141-2144.

SILVIA, P. J.; MARTIN, C.; \& NUSBAUM, E. C. (2009). A snapshot of creativity: Evaluating a quick and simple method for assessing divergent thinking. Thinking Skills and Creativity, 4(2), 79-85.

SMITH, R. E.; \& YANG, X. (2004). Toward a General Theory of Creativity in Advertising: Examining the Role of Divergence. Marketing Theory, 4(1-2), 31-58.

SMYTH, J. D.; DILlMAN, D. A., CHRISTIAN, L. M., \& MCBRIDE, M. (2009). Open-Ended Questions in Web Surveys. Public Opinion Quarterly, 73(2), 325-337.

SOLER, V. (2011). Comparative and Contrastive Observations on Scientific Titles Written in English and Spanish. English for Specific Purposes, 30(2), 124-137.

SPENCE, P. R.; LACHLAN, K. A.; \& RAINEAR, A. M. (2016). Social media and crisis research: Data collection and directions. Computers in Human Behavior, 54, $667-672$.

STIBUREK, D. (2016). Sequential testing of hypotheses about drift for Gaussian diffusions. Statistical Methodology, 33, 14-30.

STIGLER, G. J. (1995). The Nature and Role of Originality in Scientific Progress. Economica, 22(88), 293-302.

TING, C.-Y.; \& HO, C. C. (2015). Dataset of scientific inquiry learning environment. British Journal of Educational Technology, 46(5), 1038-1050.

TORRANCE, M.; THOMAS, G. V.; \& ROBINSON, E. J. (1993). Training in thesis writing: an evaluation of three conceptual orientations. British Journal of Educational Psychology, 63(1), 170-184.

TOSH, S. (2015). Checklist for procedures. Journal scan, 23(3), 13.

TURCOTTE, C.; DROLET, P.; \& GIRARD, M. (2004). Study design, originality and overall consistency influence acceptance or rejection of manuscripts submitted to the Journal. Canadian Journal of Anesthesia, 51(6), 549-556.

UIDHIR, C. M.; \& FREELAND, C. (2015). The Role of Luck in Originality and Creativity. Journal of Aesthetics and Art Criticism, 73(1), 31-55.

WALKER, D.; \& MYRICK, F. (2006). Grounded Theory, an Exploration of Process and Procedure. Qualitative Health Research, 16, 547-559.

WATTS, J. H. (2008). Challenges of supervising part-time PhD students: towards studentcentred practice. Teaching in Higher Education, 13(3), 369-373.

WEBSTER, J.; \& WATSON, R. T. (2002). Analyzing the Past to Prepare for the Future: Writing a Literature Review. MIS Quarterly, 2, 13-23. 
WEBSTER, R. (2015). The classroom experiences of pupils with special educational needs in mainstream primary schools - 1976 to 2012. What do data from systematic observation studies reveal about pupils' educational experiences over time? British Educational Research Journal, 41(6), 992-1009.

WOOD, A. M.; \& TARRIER, N. (2010). Positive Clinical Psychology: A new vision and strategy for integrated research and practice. Clinical Psychology Review, 30(7), 819-829.

ZHONG, J.; \& JIANG, S. (2016). Institutional Repositories in Chinese Open Access Development: Status, Progress, and Challenges. The Journal of Academic Librarianship, 42(6), 739-744.

ZUBER-SKERRITT, O.; \& FLETCHER, M. (2007). The quality of an action research thesis in the social sciences. Quality Assurance in Education, 15(4), 413-436.

Texto recebido em 12 de abril de 2018.

Texto aprovado em 18 de maio de 2018. 